\title{
Antineutrophil Cytoplasmic Autoantibody-Associated Vasculitis with Kidney Involvement in a Patient with AL Amyloidosis
}

\author{
Brendan L. Thoms ${ }^{a} \quad$ Varun Agrawal $^{b} \quad$ Elvira R. Umyarova ${ }^{c}$ \\ Pamela C. Gibson ${ }^{d}$ Richard J. Solomon ${ }^{b}$ \\ aDivision of Rheumatology and Clinical Immunology, University of Vermont Medical Center, \\ Burlington, VT, USA; 'bivision of Nephrology, University of Vermont Medical Center, \\ Burlington, VT, USA; 'Division of Hematology and Oncology, University of Vermont Medical \\ Center, Burlington, VT, USA; ${ }^{\mathrm{d} D e p a r t m e n t}$ of Pathology and Laboratory Medicine, University \\ of Vermont Medical Center, Burlington, VT, USA
}

Keywords

Amyloid AA and AL · Amyloidosis - ANCA-related nephritis and vasculitis ·

Glomerulonephritis · Vasculitis

\begin{abstract}
Antineutrophil cytoplasmic autoantibody (ANCA) vasculitis has occasionally been associated with other systemic glomerulonephritis, such as anti-glomerular basement membrane disease. Here, we report the first clinical case of ANCA-associated crescentic glomerulonephritis with AL amyloidosis. An 81-years-old gentleman presented to the hospital with acute kidney injury (serum creatinine $4.7 \mathrm{mg} / \mathrm{dL}$ ) on a background of chronic kidney disease and volume overload. Autoimmune serology was remarkable for p-ANCA and myeloperoxidase positivity. A renal biopsy confirmed pauci-immune glomerulonephritis and lambda light-chain amyloid deposition (confirmed on liquid chromatography and tandem mass spectrometry). The patient was initially managed with rituximab and subsequently transitioned to bortezomibbased chemotherapy but died due to decompensated heart failure. This case report promotes greater awareness of the unusual presentation of amyloidosis and guides future research and treatment.
\end{abstract}




\section{Introduction}

Amyloidosis is a disease resulting from the extracellular deposition of insoluble proteins, which aggregate into a $\beta$-pleated sheet configuration and infiltrate various organs. This is an uncommon disease, the incidence of which is 10 persons per million and can be further subdivided based on the type of protein involved [1]. AL amyloidosis is the most common form of amyloidosis and a systemic disease characterized by the expression of an amyloidogenic light chain from a plasma cell clone. Renal infiltration in amyloidosis is very common (74\%), and this is typically seen in the glomeruli, interstitial space, and extraglomerular vessels [2]. The report of glomerular crescents in patients with renal amyloid is rare and most commonly seen in AA amyloidosis rather than AL amyloidosis [3-5]. The first clinical report of crescentic glomerulonephritis reported in a patient with AL amyloidosis and multiple myeloma was in 2010 [6]. Here, we report a clinical case of antineutrophil cytoplasmic autoantibody (ANCA)associated glomerulonephritis with AL amyloidosis.

\section{Case Report/Case Presentation}

An 81-years-old Caucasian gentleman with a past medical history of stage 4 chronic kidney disease, hyperlipidemia, diastolic heart failure, benign prostate hypertrophy, and iron deficiency anemia was admitted to hospital with a 2-month history of increased urinary frequency, progressively worsening exertional dyspnea, paroxysmal nocturnal dyspnea, and peripheral edema. Symptoms associated with a 6-month history of constitutional symptoms (generalized weakness, fatigue, and poor appetite) and weight loss (10lb's).

On examination, the patient was hemodynamically stable: temperature $37.3^{\circ} \mathrm{C}$, heart rate 73 , respiratory rate 18 , blood pressure $143 / 49$, and saturating $100 \%$ on room air. Physical examination was remarkable for elevated jugular venous pressure and bilateral pitting peripheral edema. He was found to have serum creatinine of $4.7 \mathrm{mg} / \mathrm{dL}$ (baseline $2.6 \mathrm{mg} / \mathrm{dL}$ ), hemoglobin $8.8 \mathrm{~g} / \mathrm{dL}$, serum albumin $3.1 \mathrm{~g} / \mathrm{dL}$, nephrotic range proteinuria (24-h urine protein of $4 \mathrm{~g}$ ) with urine albumin/Cr ratio of $>1,890$, and microscopic hematuria (3+). Urine microscopy identified few coarsely granular casts and 3-10/HPF red blood cells. Antinuclear antibody and anti-dsDNA negative. Complement studies were with normal limits. The patient was perinuclear ANCA pattern positive, myeloperoxidase (MPO) antibody-positive (>8 $\mathrm{U}$ ), and proteinase 3 (PR3) Ab negative. Serum protein electrophoresis was negative for monoclonal immunoglobulins, and 24-h urine protein electrophoresis was negative. Analysis of serum-free light-chain ratio was normal (kappa/lambda ratio 0.66), but levels were significantly elevated consistent with the severity of his renal dysfunction (kappa and lambda free light chains ( 8.09 and $12.27 \mathrm{mg} / \mathrm{dL}$, respectively). Following a negative infective screen (Hepatitis B \& C serology, HIV and IFN $\gamma$ release assay), the patient was initially managed with rituximab $\left(375 \mathrm{mg} / \mathrm{m}^{2} \times 1\right)$, methylprednisolone $250 \mathrm{mg}$ IV daily $(\times 3)$ and subsequently transitioned to prednisone on the assumption that this was pauci-immune glomerulonephritis.

The patient underwent a kidney biopsy to confirm diagnosis and guide prognosis. A core kidney biopsy reported active focal segmental necrotizing glomerulonephritis with arteritis consistent with a pauci-immune glomerulonephritis/vasculitis. In addition, there was noted to be a background of enlarged glomeruli and extraglomerular vessels with deposition of pale eosinophilic material, which was Congo red positive, supporting the diagnosis of amyloidosis. This was seen in the kidney parenchyma and the vessels in the adipose tissue (shown in Fig. 1,2). Immunofluorescence showed the amyloid to be staining with anti-lambda only and no evidence of immune complex disease. Characteristic ultrastructural features of amyloid were seen on electron microscopy (shown in Fig. 3). Lambda amyloid was confirmed by liquid

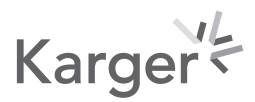



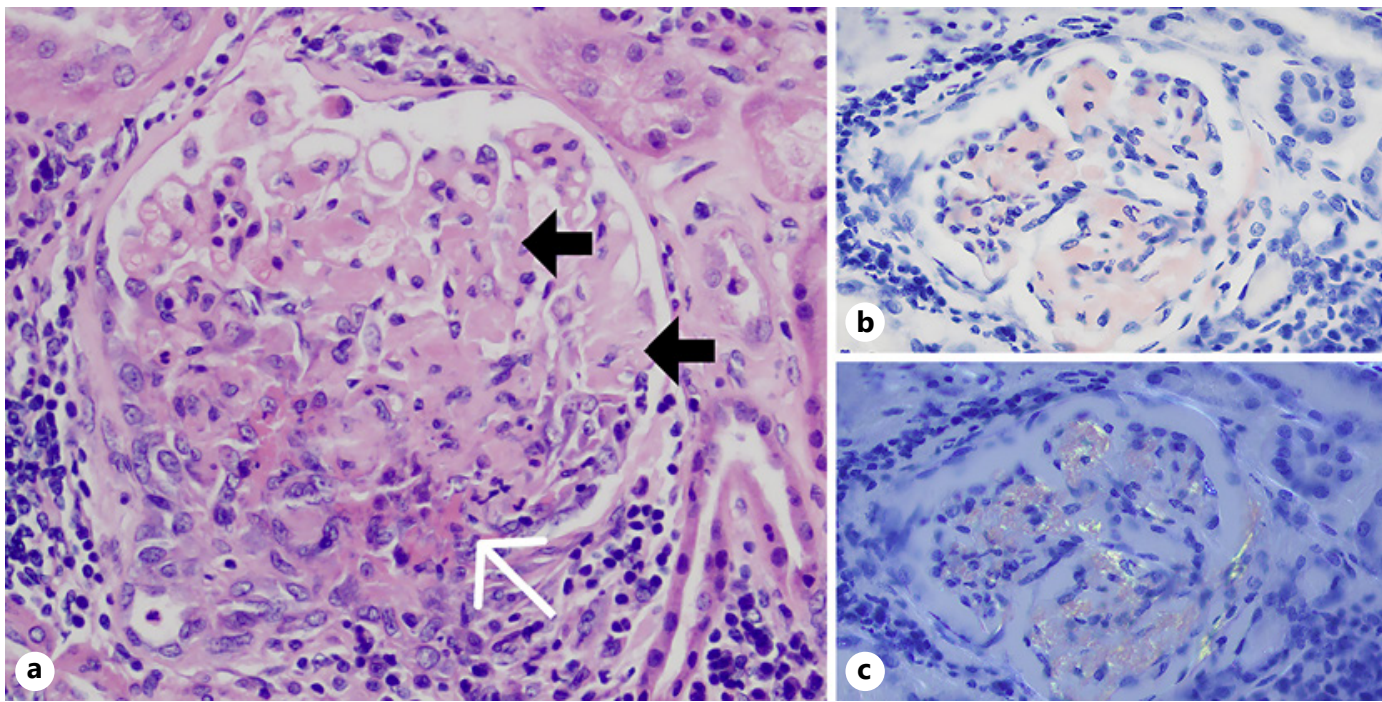

Fig. 1. a Representative glomerulus on H\&E with segmental necrosis (arrow) with thickened eosinophilic capillaries and mesangium (arrowheads) (original mag. $\times 200$ ). b, c Glomerulus stained with Congo Red under bright light (b) and polarized light (c) (original mag. $\times 400$ ).

Fig. 2. a Representative glomerulus stained with Jones Silver Stain with segmental necrosis (arrow) with thickened eosinophilic capillaries and mesangium (arrowheads) (original mag. $\times 200$ ).

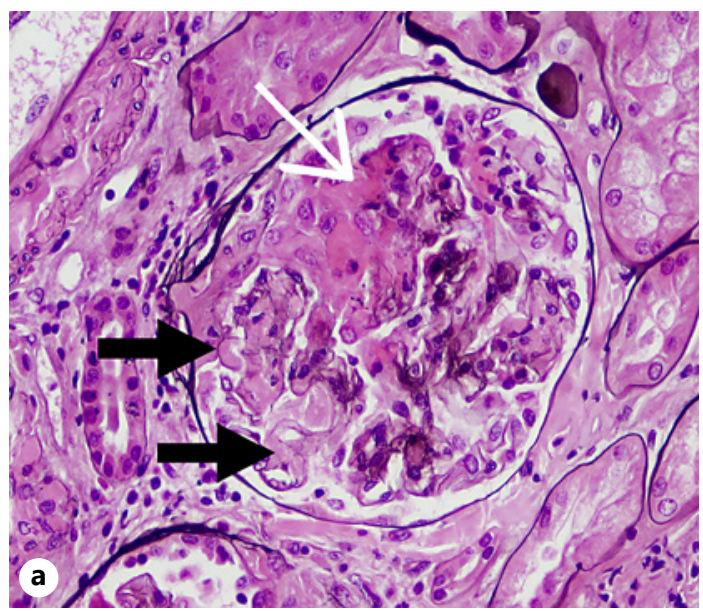

chromatography and tandem mass spectrometry, further supporting the diagnosis of AL amyloidosis (lambda-type). No clinical evidence of extrarenal vasculitis or coexisting autoimmune processes (rheumatoid arthritis, inflammatory bowel disease, or familial Mediterranean fever) was identified.

The patient underwent a CT-abdomen pelvis with and without contrast, which reported pulmonary edema, small bilateral pleural effusions, diffuse body wall edema, with no evidence of hepatosplenomegaly. Troponin 0.225 and BNP 32,500 were noted to be elevated suggesting likely cardiac amyloid involvement. A transthoracic echo reported high diastolic filling pressures, mild left ventricular hypertrophy, ejection fraction $55-60 \%$ with no regional wall motion abnormalities, and normal right ventricular size/function. A skeletal survey reported multiple suspected lytic bone lesions (proximal left tibia, distal left humerus, vertebral body $\mathrm{T} 5$, right anterior 6th rib, and right iliac crest) and vertebral body T5 compression fracture.

The patient underwent a bone marrow biopsy, which reported a normal to mildly hypocellular bone marrow with amyloidosis and decreased erythropoiesis. Staining with CD138 


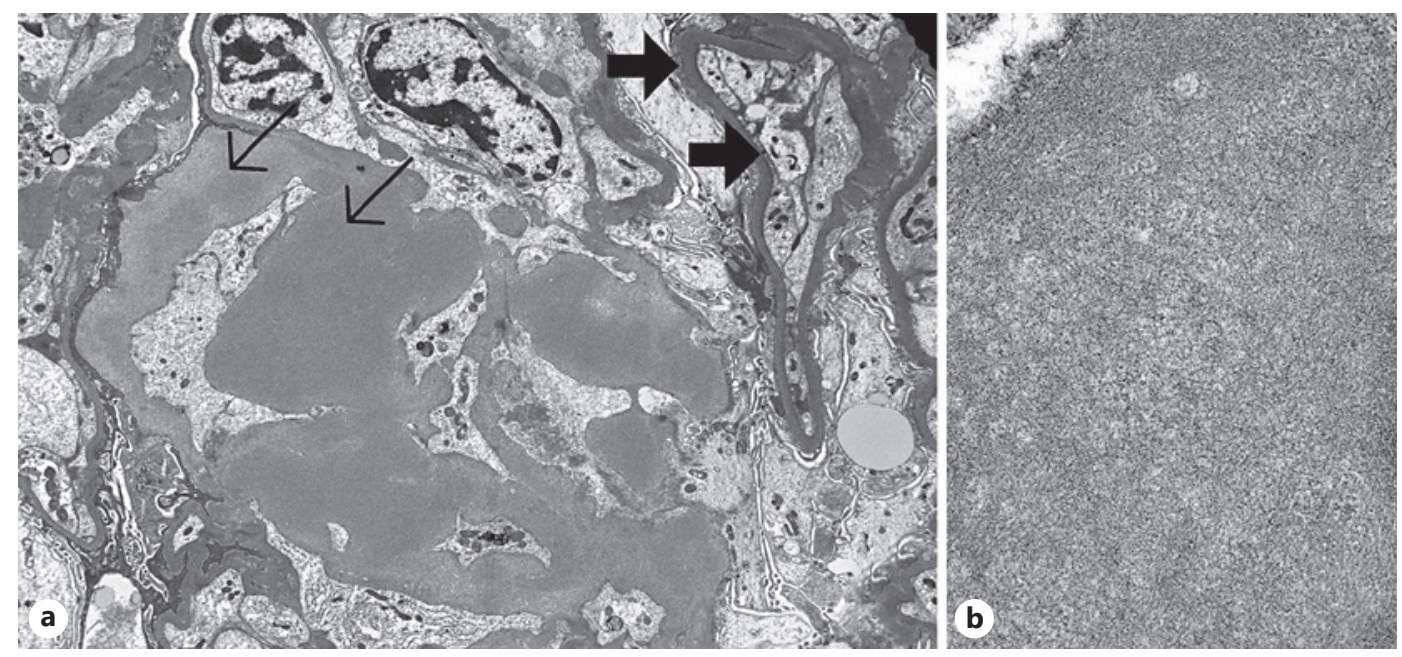

Fig. 3. a Electron microscopic image of glomerulus with amyloid deposition (arrows) and more normal regions of glomerular basement membranes for comparison (arrowheads) (original magnification $\times 1,200$ ). b Electron microscopic image of randomly arranged fibrils in glomerular basement membrane with average diameter of $9.20 \mathrm{~nm}$ (original magnification $\times 15,000$ ).

highlighted a small plasma cell population $(<3 \%)$ and but no aggregates of plasma cells. Flow cytometry showed no immunophenotypic abnormalities.

Serum creatinine improved to $3.2 \mathrm{mg} / \mathrm{dL}$ 13-days following hospitalization. Further courses of rituximab were held. The patient was discharged home with outpatient hematology follow-up. A bortezomib-based chemotherapy was initiated, and the patient completed 2 cycles. Unfortunately, the patient passed way 3-months later due to acute hypoxic respiratory failure secondary to decompensated heart failure.

\section{Discussion and Conclusion}

Renal infiltration is the most common manifestation of AL amyloidosis and this includes AL-amyloid deposition within the glomeruli, vessels, and interstitial space. Light-chain deposition results in proteinuria and chronic decline of renal function. The lambda light-chains are more amyloidogenic than kappa light chains [7]. Coexisting renal amyloidosis with crescentic glomerulonephritis is extremely rare and not recognized to be the traditional manifestation of the either disease. Crescentic glomerulonephritis with AA amyloidosis was first described by Panner at colleagues [3] and has since been reported in patients with rheumatoid arthritis and solid organ malignancies (renal cell carcinoma and cervical squamous cell carcinoma) $[3,5,8,9]$.

In 2008, retrospective work by Nagata and colleagues [10] reviewed 105 patients with renal amyloidosis (61 autopsy specimens and 44 biopsy specimens) and identified glomerular crescents in $13.3 \%$ of patients (14/105). 12/14 patients were associated with AA amyloidosis, 11 of these patients had a background of rheumatoid arthritis, and 1 patient had a background of macroglobulinemia. 2/14 specimens were associated with AL $(\lambda)$ amyloidosis (one with plasma cell dysplasia and one with multiple myeloma). Nagata and colleagues [10] proposed that the prevalence of crescentic glomerulonephritis associated with amyloidosis may be more common than previously thought.

Crosthwaite and colleagues [6] described the first clinical report of crescentic glomerulonephritis reported in a patient with AL amyloidosis and IgGк multiple myeloma. Auto-

\section{Karger'}


immune serology was negative, with no evidence of coexisting vasculitis. Despite the treatment of multiple myeloma, the patient progressed to end-stage kidney disease and passed away.

The pathogenesis of concurrent renal amyloidosis with crescentic glomerulonephritis is not well understood. It has previously been suggested that these are 2 distinct processes [11]. Nagata and colleagues [10] identified a correlation between amyloid localization and crescent formation on light microscopy. Additionally, there was noted to be disruption of the glomerular basement membrane by amyloid fibrils on electron microscopy. Nagata and colleagues [10] hypothesized explanations include basement membrane disruption leading to the escape of fibrin into the Bowman's space provoking crescent formation or amyloid itself being proinflammatory leading to glomerular inflammation.

Here, we report the first clinical case of pauci-immune glomerulonephritis secondary to kidney limited ANCA vasculitis in association with AL amyloidosis. The biopsy findings support the clinical impression of acute kidney injury superimposed on chronic kidney disease secondary to MPO + ANCA-associated vasculitis without clinical evidence of extrarenal involvement. Regarding pathophysiology, we propose that these 2 entities could be related or distinct:

- Our first explanation is that these 2 processes were likely distinct diseases. This is supported by the patient's CKD stage 4, diastolic heart failure and elevated BNP suggesting long-standing AL amyloidosis over many months or possibly years. At the time of hospital admission, the patient had experienced a rapid deterioration in kidney function on prior chronic kidney disease, with evidence of necrosis on biopsy and slight renal function recovery in response to immunosuppressive therapy. This is suggestive of rapidly progressive ANCA-associated glomerulonephritis on a background of AL amyloidosis. Given the uncommon incidences of both disorders (AL amyloidosis 9.7 cases per million person-years in US adults and pauci-immune glomerulonephritis 3.1 cases per million person-years in US adults), the likelihood of a patient having both disorders is approximately 1 in 30 billion [12,13].

- Our second explanation is that the pauci-immune vasculitis occurred due to chronic AL amyloidosis. We hypothesize that vascular and perivascular amyloid deposition within the small vessels of the kidney and glomerulus predisposes neutrophils to mechanical rupture, exposing new, previously cytosolic/perinuclear epitopes (e.g., MPO) and the developmentofautoantibodies.Amyloid co-localizationwithcrescenticglomerulonephritis is confounded by the ubiquitous infiltration of amyloid within the kidney. Amyloidinduced kidney damage could also result in neoepitope formation. This neoepitope formation, however, is unlikely to be MPO. MPO is predominantly expressed by neutrophils, monocytes/macrophages and is not endogenously expressed by renal tissue [14]. Increased expression of tumor necrosis factor-alpha (TNF- $\alpha$ ) has been previously reported to "prime" neutrophils to enhance the expression of autoantibody promoting epitopes. However, AL-amyloidosis is considered a noninflammatory infiltrative disease. There is no evidence of increased TNF- $\alpha$ expression, and prior trials of etanercept (a soluble receptor that binds TNF- $\alpha$ ) in patients with AL-Amyloidosis identified no significant difference in median survival [15].

A skeletal survey of our patient reported multiple suspected lytic bony lesions. Serum protein electrophoresis was negative for monoclonal immunoglobulins, and 24-h urine protein electrophoresis was negative arguing against multiple myeloma. Additionally, multiple myeloma was not identified on bone marrow biopsy. Lytic lesions are not classically associated with AL amyloidosis but have been previously reported in the literature. The extracellular deposition of amyloidosis in bone is thought to lead to destruction mimicking lytic lesions in the absence of multiple myeloma $[16,17]$.

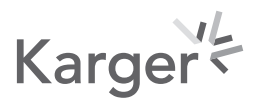


Case Reports

in Nephrology

and Dialysis

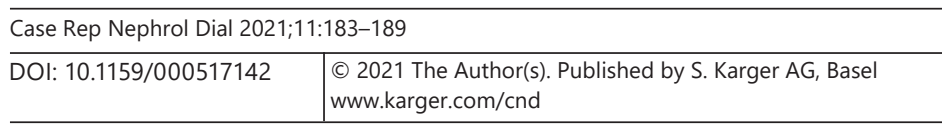

Thoms et al.: ANCA-Associated Vasculitis in a Patient with AL Amyloidosis

The patient passed away of acute hypoxic respiratory failure in the setting of decompensated heart failure secondary. It is important to note that he received 2 cycles of bortezomibbased chemotherapy before his decompensation as this medication has been associated with acute exacerbation of preexisting heart failure [18].

In summary, ANCA-associated glomerulonephritis with AL amyloidosis is rarely observed in patients. Given the patient's prior chronic kidney disease, diastolic heart failure and elevated BNP the patient likely developed ANCA-associated glomerulonephritis on a background of AL amyloidosis. The underlying basis for the association remains unclear. Given the low likelihood of these rare diseases occurring in the same patient as distinct processes, we propose vascular amyloid deposition may predispose to neutrophil rupture and neoepitope formation as a possible explanation as how these diseases may be related. Our case is consistent with limited examples in the literature that suggest that this presentation reflects a poor patient prognosis. We hope that greater awareness will guide future research and treatment.

\section{Acknowledgement}

In the acknowledgement section, authors must include individuals and organizations that have made substantive contributions to the research or the manuscript. An exception is where funding was provided, which should be included in funding sources. Please refer to the guidelines issued by the ICMJE to determine nonauthor contributors that should be included in the acknowledgement section.

\section{Statement of Ethics}

A case report is a medical/educational activity that does not meet the Department of Health and Human Services definition of "research," which is: "a systematic investigation, including research development, testing, and evaluation, designed to develop or contribute to generalizable knowledge." Therefore, the paper is exempt from Ethical Committee approval. Informed written consent was obtained from the patient's next of kin.

\section{Conflict of Interest Statement}

The authors have no conflicts of interest to declare.

\section{Funding Sources}

No sources of funding to disclose.

\section{Author Contributions}

Contribution of the authors is as follows: R.J.S., B.L.T., and E.R.U. treated the patient. V.A. consulted on the patient. P.C.G. diagnosed the patient's pathology. 


\section{References}

1 Sipe JD, Benson MD, Buxbaum JN, Ikeda S, Merlini G, Saraiva MJ, et al. Nomenclature 2014: amyloid fibril proteins and clinical classification of the amyloidosis. Amyloid. 2014 Dec;21(4):221-4.

2 Kyle RA, Linos A, Beard CM, Linke RP, Gertz MA, O'Fallon WM, et al. Incidence and natural history of primary systemic amyloidosis in Olmsted County, Minnesota, 1950 through 1989. Blood. 1992 Apr;79(7):1817-22.

3 Panner BJ. Rapidly progressive glomerulonephritis and possible amyloidosis. Arch Pathol Lab Med. 1980 Nov; 104(11):603-9.

4 Watanabe T, Toyoshima H, Nagafuchi Y, Yoshikawa Y. Renal amyloidosis with crescents. Hum Pathol. 1984 Jul; 15(7):684-6.

5 Harada A, Tomita Y, Yamamoto H, Onoyama K, Omae T, Oh Y, et al. Renal amyloidosis associated with crescentic glomerulonephritis. Am J Nephrol. 1984;4(1):52-5.

6 Crosthwaite A, Skene A, Mount P. Rapidly progressive glomerulonephritis complicating primary AL amyloidosis and multiple myeloma. Nephrol Dial Transplant. 2010 Aug;25(8):2786-9.

7 Glenner GG. Amyloid deposits and amyloidosis. The beta-fibrilloses (first of two parts). N Engl J Med. 1980 Jun; 302(23):1283-92.

8 Schafernak KT, Chugh SS, Kanwar YS. Co-existent crescentic glomerulonephritis and renal amyloidosis: a case report and literature review. J Nephrol. 2005 Sep-Oct;18(5):616-22.

9 Zuckerman JE, Peng F, Karl BE, Schulze CE, Sisk A. Cancer-associated AA amyloidosis presenting as crescentic glomerulonephritis. Kidney Int Rep. 2019 Jun;4(6):882-7.

10 Nagata M, Shimokama T, Harada A, Koyama A, Watanabe T. Glomerular crescents in renal amyloidosis: an epiphenomenon or distinct pathology? Pathol Int. 2001 Mar;51(3):179-86.

11 Bernheim J. The patient with two renal diseases: crescentic glomerulonephritis and renal AA amyloid. Nephrol Dial Transplant. 1999 May;14(5):1315-6.

12 Quock TP, Yan T, Chang E, Guthrie S, Broder MS. Epidemiology of AL amyloidosis: a real-world study using US claims data. Blood Adv. 2018 May;2(10):1046-53.

13 Pettersson EE, Sundelin B, Heigl Z. Incidence and outcome of pauci-immune necrotizing and crescentic glomerulonephritis in adults. Clin Nephrol. 1995 Mar;43(3):141-9.

14 Sanders JS, van Goor H, Hanemaaijer R, Kallenberg CG, Stegeman CA. Renal expression of matrix metalloproteinases in human ANCA-associated glomerulonephritis. Nephrol Dial Transplant. 2004 Jun;19(6):1412-9.

15 Hussein MA, Juturi JV, Rybicki L, Lutton S, Murphy BR, Karam MA. Etanercept therapy in patients with advanced primary amyloidosis. Med Oncol. 2003;20(3):283-90.

16 Kramer MR, van Dijk JM, Hadas I, Hershko C. Destructive bone lesions in primary amyloidosis. Postgrad Med J. 1986 Nov;62(733):1037-41.

17 Schonland SO, Hansmann J, Mechtersheimer G, Goldschmidt H, Ho AD, Hegenbart U. Bone involvement in patients with systemic AL amyloidosis mimics lytic myeloma bone disease. Haematologica. 2008 Jun;93(6): 955-6.

18 Cornell RF, Ky B, Weiss BM, Dahm CN, Gupta DK, Du L, et al. Prospective study of cardiac events during proteasome inhibitor therapy for relapsed multiple myeloma. J Clin Oncol. 2019 Aug;37 (22):1946-55. 